\title{
Cell therapies: why scale matters
}

\author{
"As cell therapies edge closer to commercial scale, there is a pressing \\ need to understand the implications of scale up and what effect it \\ will have on the cell therapy manufacturing process."
}

\begin{abstract}
Keywords: bioprocessing • bioreactor $\bullet$ cell therapy $\bullet$ microcarrier $\bullet$ regenerative medicine - scale up • small-scale
\end{abstract}

Cell therapies provide a novel treatment option with the ability to replace, repair or regenerate damaged cells and tissue and have the potential to change the way in which medicine is practiced [1]. Unlike traditional biopharmaceutical manufacture where the cell is used as a factory for the production of proteins or attenuated viruses, cell therapy products comprise of live fully functional cells, which form the basis of the treatment. As such, the development of efficacious cell therapies presents new challenges whereby biomanufacturing processes need to account for cell quality from the point of isolation through expansion, recovery, formulation and final delivery to the patient. This does not rely solely on furthering our biological understanding of the cell, but also incorporates significant engineering and biomanufacturing hurdles, which must be simultaneously overcome. At present, there are over 200 clinical trials involving human mesenchymal stem cells (hMSCs) alone, the majority of which are in early stages of clinical trials (Phase I and Phase II), and it is recognized that in order to meet the clinical demand for cells of sufficient quality and quantity, smallscale processes for many cell therapy candidates require a step change in the design of their corresponding manufacturing systems.

While the scientific potential of cell therapies and tissue-engineered regenerative medicine products has been demonstrated (e.g., tissue-engineered trachea [2]), the industry has been hampered by limited commercial success and modest clinical uptake resulting primarily from difficulties in establishing clinical utility and cost-effectiveness [3]. While the former is currently being addressed by companies targeting 'first-in-man' studies, the latter can only be achieved by improving our process and product understanding such that cell therapy products can be manufactured at a suitable scale and at a price that the relevant reimbursement agencies are willing to bare.

It would therefore seem obvious to many why cell therapy processes need to be scaled, yet seemingly less obvious are the engineering and the related biological difficulties associated with large-scale production. As with early biopharmaceutical production, the modus operandi for many cell therapy candidates currently in development is that scalability is usually an afterthought and normally the final step in any $R \& D$ program. There is a tendency to assume that given a process operates efficiently at the laboratory scale, it is a relatively trivial matter to achieve the same level of efficiency and productivity at the industrial or production scale. However this is not the case with many process engineering considerations needing to be taken into account, it is not simple matter of 'making the pot bigger' and there is often a detrimental effect on bioprocess performance as we move up through the scales $[4,5]$. Many cell therapies currently under development are based on manual T-flaskbased processes, which will have to be drastically redesigned to achieve the required cell number to meet predicted clinical demand. Even with autologous cell therapies where a scale-out approach is likely to be employed, the challenge of manipulating and processing multiple units in a closed, sterile manner can

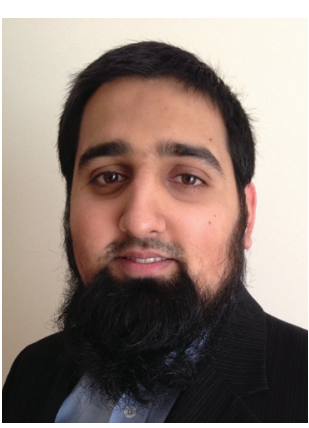

Qasim A Rafiq Aston Medical Research Institute, School of Life \& Health Sciences, Aston University, Aston Triangle, Birmingham, B4 7ET, UK and Centre for Biological Engineering, Loughborough University, Leicestershire, LE11 3TU, UK

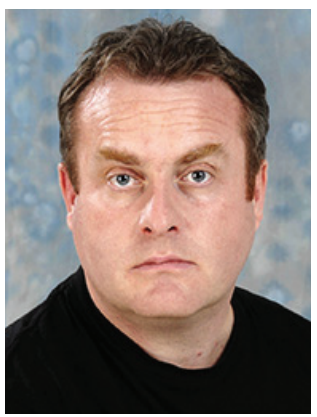

Christopher J Hewitt Author for correspondence: Aston Medical Research Institute, School of Life \& Health Sciences, Aston University, Aston Triangle, Birmingham, B4 7ET, UK and Centre for Biological Engineering, Loughborough University, Leicestershire, LE11 3TU, UK Tel.: +44 (0)1212044949 c.j.hewitt@aston.ac.uk

FUTURE SCIENCE partof 
be extremely difficult. Routine processing steps such as passaging cells, performing medium exchanges and the sampling of cell culture broth require significant personnel and resources, or investment in automated manufacturing technologies which in turn involves significant capital expenditure and will require comparability studies between both the manual and automated processes before being accepted by the regulator.

\section{"Efforts are now being made in the biopharmaceutical industry to use relevant experimental scale-down models, which are representative of larger-scale industrial systems..."}

For allogeneic cell therapy processes scale-up approaches are more likely to be employed, however, the challenges are even more significant from a cell expansion perspective. The use of stirred-tank bioreactors for the scale up of biopharmaceuticals is mostly routine, however given that most cell therapy candidates employ anchorage-dependent cells, microcarriers are required for stirred-tank bioreactor expansion. Moving from a manual, T-flask-based process to a microcarrier stirredtank bioreactor process is a substantial transition, which requires significant process and product understanding. Furthermore, harvesting the cells from the microcarriers while maintaining the cells' critical to quality attributes is very different from monolayer culture and poses a significant challenge, but work has begun to develop a scalable recovery step [6]. Indeed, we recently published the first peer-reviewed study of hMSC expansion and recovery from microcarriers in a stirred-tank bioreactor at the 5-1 scale (2.5-1 working volume) [7], however for industrial scale processes, it is likely that the scale will need to be between 500 and 25001 to achieve the predicted cell numbers required $[8,9]$. In essence, the objective of scaling up any bioprocess is to overcome the design conditions and operational procedures, such that during translation to the larger scale, the optimal environmental conditions for the bioprocess (as identified at the small scale) are maintained. These environmental conditions include appropriate nutrient and growth inhibiting metabolite concentrations as well as maintaining in vivo $\mathrm{pH}$, temperature, dissolved oxygen and carbon dioxide concentrations.

Much can be learned from traditional bioprocessing scale up. It has often been found that for both microbial and mammalian aerobic cultures, biomass yield and the production of growth-associated pharmaceutical products tend to decrease on scale up [4,10-12]. Such poor performance at the large scale is known to be due to the presence of spatial and temporal concentration gradients arising from longer mixing times and poorer mixing generally in comparison to the small scale when a fed-batch strategy (preferred by industry) is employed with $\mathrm{pH}$ control by automatic titration $[4,5,13,14]$. For cell therapies, where cell quality is of paramount importance, any fluctuations in the culture environment are likely to impact the product's critical to quality attributes. As such, there needs to be a greater level of attention paid to the issues that will arise during scale up, with a focus on the engineering characterization and process comparability. In particular, where large-scale bioreactors are being used $\left(>0.5 \mathrm{~m}^{3}\right)$, significant effort must be made to understand and characterize the mixing environment within the vessel and identify any potential issues early on in development. It is highly probable that temporal and spatial gradients will indeed exist since a perfusion strategy is the likely way forward. Therefore the effects of cells circulating through such gradients on their quantity, quality and viability will need to be quantified and mechanisms of mitigating against these effects, if any, identified. Furthermore, to aid the transition through scale up, the development of relevant experimental scale-down models should be a priority, as this will ensure significant expenditure and resource is not wasted only to find that the process performs poorly at the large scale. A novel experimental model for mimicking the poorly mixed conditions at the large scale has been devised [4] by using a smallscale stirred-tank bioreactor connected in series with a plug-flow bioreactor (used to mimic the addition zone for nutrient feeds and $\mathrm{pH}$ controlling agents at the large scale). This model was found to be more representative of large-scale bioreactor performance with respect to the required bioprocessing outcomes than the small-scale stirred-tank bioreactor in isolation.

Efforts are now being made in the biopharmaceutical industry to use relevant experimental scale-down models, which are representative of larger-scale industrial systems $[10,15]$ and which have proven to be successful with respect to screening protein-expressing clones as well as for the optimization of culture conditions and feeding regimes for optimal cell yield and product titer [16]. Until now, small-scale models have primarily taken place in 50-ml nonsparged shake flasks [4], however large-scale industrial processes usually take place in the controlled environment of a stirred-tank bioreactor. As a result, bioperformance between the small-scale shake flask and the large-scale stirred-tank bioreactor often differs, with high producing clones taken forward based on small-scale studies which do not perform as expected at the large scale [16]. It has been demonstrated [10] that the ambr ${ }^{\mathrm{TM}}$ (TAP Biosystems, now a Sartorius Stedim company), a high-throughput microbioreactor system (15-ml maximum working volume), performed simi- 
larly with respect to cell growth and IgG4 productivity to that achieved in a 5-1 stirred-tank bioreactor, while the 250 -ml shake flasks run in parallel were significantly different [16]. It was therefore suggested that the ambr was a better scale-down model for clonal selection than the traditional shake flask model. Similar findings have been reported by others including biopharmaceutical companies such as Genentech (CA, USA) [17], Merck (NJ, USA) [18] and MedImmune (MD, USA) [19]. It is therefore our contention that suitable small-scale models which mimic cell viability, and more importantly, cell quality at the large scale need to be developed for cell therapies which may identify significant cost savings during scale up [20]. To this end, we are currently investigating the amenability of the ambr microbioreactor system for hMSC microcarrier culture as a suitable high-throughput, scale-down model in place of $100-\mathrm{ml}$ spinner flasks and initial results appear positive.

As cell therapies edge closer to commercial scale, there is a pressing need to understand the implications of scale up and what effect it will have on the

\section{References}

1 Rowley JA. Developing cell therapy biomanufacturing processes. Chem. Eng. Prog. 106, 50-55 (2010).

2 Macchiarini P, Jungebluth P, Go T et al. Clinical transplantation of a tissue-engineered airway. Lancet 372, 2023-2030 (2008).

3 Williams DJ, Thomas RJ, Hourd PC et al. Precision manufacturing for clinical-quality regenerative medicines. Philos. Trans. A Math. Phys. Eng. Sci. 370, 3924-3949 (2012).

4 Hewitt CJ, Nienow AW. The scale-up of microbial batch and fed-batch fermentation processes. Adv. Appl. Microbiol. 62, 105-135 (2007).

5 Nienow AW. Reactor engineering in large scale animal cell culture. Cytotechnology 50, 9-33 (2006).

6 Nienow AW, Rafiq QA, Coopman K, Hewitt CJ. A potentially scalable method for the harvesting of hMSCs from microcarriers. Biochem. Eng. J. 85, 79-88 (2014).

7 Rafiq QA, Brosnan KM, Coopman K, Nienow AW, Hewitt CJ. Culture of human mesenchymal stem cells on microcarriers in a 51 stirred-tank bioreactor. Biotechnol. Lett. 35, 1233-1245 (2013).

8 Mason C, Dunnill P. Quantities of cells used for regenerative medicine and some implications for clinicians and bioprocessors. Regen. Med. 4 153-157 (2009).

9 Rafiq QA, Coopman K, Hewitt CJ. Scale-up of human mesenchymal stem cell culture: current technologies and future challenges. Curr. Opin. Chem. Eng. 2, 8-16 (2013).

10 Nienow AW, Scott WH, Hewitt CJ et al. Scale-down studies for assessing the impact of different stress parameters on growth and product quality during animal cell culture. Chem. Eng. Res. Des. 91(11), 2265-2274 (2013).

11 Enfors SO, Jahic M, Rozkov A et al. Physiological responses to mixing in large scale bioreactors. J. Biotechnol. 85, 175-185 (2001). cell therapy manufacturing process. These products rely on stringent processing conditions throughout, as the cells expanded at this scale are the very same cells that will be administered to patients. No longer should scale up be left to the end, but rather scalability should be considered early on in the development process and strategies should be in place from the outset to allow for a smooth transition from small-scale production to larger, clinical-scale manufacturing. If not, we may end up repeating the mistakes of the past and redesigning processes all over again.

\section{Financial \& competing interests disclosure}

The authors have no relevant affiliations or financial involvement with any organization or entity with a financial interest in or financial conflict with the subject matter or materials discussed in the manuscript. This includes employment, consultancies, honoraria, stock ownership or options, expert testimony, grants or patents received or pending or royalties.

No writing assistance was utilized in the production of this manuscript.

12 George S, Larsson G, Enfors SO. A scale-down twocompartment reactor with controlled substrate oscillations: metabolic response of Saccharomyces cerevisiae. Bioprocess Eng. 9, 249-257 (1993).

13 Bylund F, Collet E, Enfors SO, Larsson G. Substrate gradient formation in the large-scale bioreactor lowers cell yield and increases by-product formation. Bioprocess. Eng. 18, 171-180 (1998).

14 Enfors SO. Preface. In: Advances in Biochemical Engineering/ Biotechnology - Physiological Stress Responses in Bioprocesses Volume 89. Enfors SO (Ed.). Springer, NY, USA, IX-X (2004).

15 Pollard D. Are automated disposable small-scale reactors set to dominate the future of pharmaceutical bioprocess development? Pharm. Bioprocess. 2, 9-12 (2014).

16 Nienow AW, Rielly CD, Brosnan K et al. The physical characterisation of a microscale parallel bioreactor platform with an industrial $\mathrm{CHO}$ cell line expressing an IgG4. Biochem. Eng. J. 76, 25-36 (2013).

17 Hsu WT, Aulakh RP, Traul DL, Yuk IH. Advanced microscale bioreactor system: a representative scale-down model for bench-top bioreactors. Cytotechnology 64, 667-678 (2012).

18 Moses S, Manahan M, Ambrogelly A, Ling WLW. Assessment of AMBRTM as a model for high-throughput cell culture process development strategy. Adv. Biosci. Biotechnol. 3, 918 (2012).

19 Lewis G, Lugg R, Lee K, Wales R. Novel automated microscale bioreactor technology: a qualitative and quantitative mimic for early process development. Bioprocess. J. 9, 22-25 (2010).

20 Rafiq QA, Coopman K, Nienow A.W, Hewitt CJ. A quantitative approach for understanding small-scale human mesenchymal stem cell culture - implications for large-scale bioprocess development. Biotechnol. J. 8, 459-471 (2013). 